\title{
Erratum to: Outcomes of abdominal radical trachelectomy: results of a multicenter prospective cohort study in a Tohoku Gynecologic Cancer Unit
}

\author{
Hideki Tokunaga $\cdot$ Yoh Watanabe $\cdot$ Hitoshi Niikura $\cdot$ Satoru Nagase $\cdot$ Masafumi Toyoshima $\cdot$ \\ Reiji Shiro $\cdot$ Yoshihito Yokoyama $\cdot$ Hideki Mizunuma $\cdot$ Tsuyoshi Ohta $\cdot$ Hiroshi Nishiyama $\cdot$ Takafumi Watanabe \\ Naoki Sato $\cdot$ Toru Sugiyama $\cdot$ Tadao Takano $\cdot$ Fumiaki Takahashi $\cdot$ Nobuo Yaegashi
}

Published online: 5 December 2014

(C) Japan Society of Clinical Oncology 2014

\section{Erratum to: Int J Clin Oncol}

DOI 10.1007/s10147-014-0763-6

The name of the 12th author should be given as Naoki Sato, not Naoto Sato.

The online version of the original article can be found under doi:10.1007/s10147-014-0763-6.

H. Tokunaga $\cdot$ Y. Watanabe $(\bowtie) \cdot H$. Niikura $\cdot$ S. Nagase $\cdot$

M. Toyoshima $\cdot$ R. Shiro $\cdot$ T. Takano $\cdot$ N. Yaegashi

Department of Obstetrics and Gynecology, Tohoku University Graduate School of Medicine, Sendai, Japan

e-mail: watanabe@med.tohoku.ac.jp

Y. Watanabe $\cdot$ T. Takano $\cdot$ F. Takahashi

Department of Clinical Research Network, Clinical Research, Innovation and Education Center, Tohoku University Hospital,

1-1 Seiryo-machi, Aoba-ku, Sendai, Miyagi 980-8574, Japan

Y. Yokoyama $\cdot$ H. Mizunuma

Department of Obstetrics and Gynecology, Hirosaki University Graduate School of Medicine, Hirosaki, Japan

T. Ohta

Department of Obstetrics and Gynecology, Yamagata University

Faculty of Medicine, Yamagata, Japan
H. Nishiyama $\cdot$ T. Watanabe

Department of Obstetrics and Gynecology, Fukushima Medical

University, Fukushima, Japan

N. Sato

Department of Obstetrics and Gynecology, Akita University

Graduate School of Medicine, Akita, Japan

T. Sugiyama

Department of Obstetrics and Gynecology, Iwate Medical

University, Morioka, Japan 